\title{
LOS CAMINOS COMPARTIDOS \\ EN LA HISTORIA DEL DERECHO \\ DE AUTOR Y EL DEPÓSITO LEGAL \\ EN EL REINO UNIDO
}

\begin{abstract}
JHONNY ANTONIO PABÓN CADAVID*
\end{abstract}
\section{INTRODUCCIÓN}

El depósito legal, desde sus inicios, ha sido un instrumento de política cultural cuyo propósito es recolectar y resguardar la producción intelectual de un país. La aparición de la cultura impresa creó interés en la colección y preservación de todas las publicaciones. El depósito legal surgió en Francia junto con el mercado de la impresión y fue la primera legislación de patrimonio cultural durante el Renacimiento ${ }^{1}$. La ordenanza de Montpellier de 28 de diciembre de 1537, emitida por el rey Francisco I, requirió que cada libro impreso fuera enviado a su biblioteca en el Château de Blois en Fontainebleau con el objetivo de conservarlo para la posteridad de la memoria de la humanidad ${ }^{2}$. En 1617, el depósito legal se convirtió

\footnotetext{
* Docente-investigador, Departamento de la Propiedad Intelectual, Universidad Externado de Colombia, Bogotá (Colombia). Ph.D. Victoria University of Wellington, Nueva Zelanda. Magíster en Historia, Pontificia Universidad Javeriana, Colombia. Magister Science en Knowledge Management, Nanyang University, Singapur. Abogado, Universidad Externado de Colombia. Miembro correspondiente de la Academia Colombiana de Historia. Investigador asociado en Le Centre d'Études et de Recherche en Droit de l’Immatériel, Université Paris Sud, Francia. Contacto: pabonjhon@myvuw.ac.nz Fecha de recepción: 14 de marzo de 2018. Fecha de aceptación: 3 de mayo de 2018. Para citar el artículo: Pabón Cadavid, J. A. "Los caminos compartidos en la historia del derecho de autor y el depósito legal en el Reino Unido", Revista La Propiedad Inmaterial n. ${ }^{\circ}$ 25, Universidad Externado de Colombia, enero-junio 2018, pp. 73-92. Dor. https://doi. org/10.18601/16571959.n25.04

1 Chastel, André y Babelon, Jean-Pierre. La notion du patrimoine, Paris, Liana Levi, 1980, p. 10.

2 Ordonnance de Montpellier du 28 décembre 1537 (France). (BNF, Ms 22076, Catalogue des Actes de François 1er, N 9476); Principaux textes sur le dépôt légal, Historique (Direction de l'Administration Générale, Paris, 1992). Para Estrid Bjerregärd, el depósito legal emergió como un instrumento de censura: "keeping control of the new and dangerous medium, the printed word. Censorship was therefore a prevailing reason behind many of the early ordinances such as the French Montpellier ordinance of 1537". Estrid BJerregärd, "Legal deposit - Purpose and scope in Modern Society", Libri, Copenaghen, 23 (4), 1973, p. 331. Otros académicos han adoptado una posición diferente; ver P. STIRLING et al., "The State of e-legal deposit in France: Looking back at five years of putting new legislation into practice and envisioning the future" (2012) 38 (1) IfLA Journal 5, p. 6; Kenneth D. Crews, "Legal deposit in four countries: Laws and library services" (1988) 80 Law Library Journal 551, p. 553.
} 
en un requisito para la concesión de privilegios ${ }^{3}$, los cuales son el antecedente de los derechos de autor. Estos derechos de propiedad intelectual, desde sus inicios, han tenido como propósito incentivar la producción intelectual, la difusión del conocimiento y la creación de mercados alrededor de lo que hoy se denominan industrias culturales o creativas.

La difusión internacional del depósito legal y del derecho de autor ha transcurrido junto con el desarrollo de las tecnologías de la información. El depósito legal se ha implementado y adaptado dentro de diversos contextos legales y políticos. Las diferencias en cada jurisdicción se derivan principalmente del momento histórico en el que surgió la obligación de entrega de ejemplares a una institución cultural, el contexto institucional y los alcances y objetivos de estas figuras legales en cada país. En Inglaterra, la reina María, en 1557, concedió el control y monopolio del mercado editorial a la Compañía de Impresores (Stationers' Company). En 1602, sir Thomas Bodley inauguró su biblioteca en la Universidad de Oxford. En 1610, dentro de los esfuerzos por enriquecer las colecciones de la biblioteca, Bodley persuadió a la Compañía de Impresores de entregar de forma gratuita copia de los libros publicados ${ }^{4}$. Este acuerdo fue ideado por Thomas James 5 , encargado de la Biblioteca Bodleiana hasta $1620^{[6]}$. James basó la idea de obtener libros publicados en el sistema francés de depósito legal7 ${ }^{7}$. El acuerdo de 1610, entre la Compañía de Impresores y Bodley, fue el precursor del depósito legal en Inglaterra. Este acuerdo surge como una medida para aumentar las colecciones académicas de la Biblioteca Bodleiana para el uso de los intelectuales, y además sirvió al interés privado de la industria editorial. Una de las principales funciones del acuerdo fue la creación de un repositorio para facilitar a la Compañía de Impresores el acceso a copias de libros que estuvieran fuera del mercado. El acuerdo estableció que, cuando un editor quisiera volver a imprimir un libro agotado o crear una versión modificada, la Compañía tenía el derecho de solicitar prestada una copia del material depositado $^{8}$. En la tradición francesa, el depósito legal no tuvo como objetivo satisfacer ningún interés de la industria editorial.

La renuencia de los editores a entregar libros fue evidente por el incumplimiento del acuerdo9. Por ejemplo, en 1613, la Biblioteca Bodleiana recibió de la Compañía de Impresores 41 impresos, y en 1614 obtuvo 72 impresos, lo cual equivale a cerca del $15 \%$ de sus publicaciones ${ }^{10}$. Con el decreto de once de julio de 1637 se

3 Marie-Thérèse Dougnac y M. Guilbaud, "Le dépôt légal: son sens et son evolution" (1960) 8 Bulletin des Bibliothèques de France.

4 R. C. Barrington Partridge, The History of the Legal Deposit of Books throughout the British Empire, The Library Association, London, 1938, p. 17.

5 Philip, IAn, The Bodleian Library in the Seventeenth \& Eighteenth Centuries, Clarendon Press, Oxford, 1983, p. 27.

6 Ibíd., p. 11.

7 BARRINGTON (1938) p. 18

8 A Deed of Grant from the Stationer's Company, entitling the Bodleian Library to the first correct impression of every book printed by them, 1610 .

9 BARRINGTON (1938) p. 21

10 Philip (1983) p. 28. 
reguló pormenorizadamente el comercio de impresos ${ }^{11}$. La Universidad de Oxford logró incluir en este decreto la obligación para los editores e impresores de entregar una copia de sus publicaciones a su biblioteca ${ }^{12}$. Como sanción por el incumplimiento en el envío de ejemplares, el decreto estableció una multa pecuniaria y el encarcelamiento $^{13}$. Sin embargo, este decreto fue derogado en 1640. La entrega de volúmenes a la Biblioteca Bodleiana volvió a ser absolutamente voluntaria, ya que el acuerdo de 1610 había perdido su validez ${ }^{14}$. Las pocas copias que recibió la Biblioteca Bodleiana durante los siguientes años se debieron a la entrega voluntaria y a los esfuerzos de la Universidad de Oxford por recopilar las obras publicadas durante la guerra civil ${ }^{15}$.

La legislación de imprenta en vigor entre 1662-1679 y 1685-1695 estableció una fuerte censura y consolidó el monopolio legal de la Compañía de Impresores en el mercado editorial ${ }^{16}$. Además, estableció la obligación para los impresores de enviar copias a las universidades más importantes de Inglaterra -Oxford y Cambridge- y una tercera copia a la Biblioteca Real. Se ha considerado que el depósito legal fue un instrumento contra la libertad de prensa al aparecer en el marco de la legislación de censura ${ }^{17}$. Sin embargo, su inclusión en esta fue una oportunidad para incorporar las solicitudes hechas desde el comienzo del siglo XVI por las universidades. El objetivo del depósito legal estuvo relacionado con la promoción de la educación y la preservación cultural mediante el enriquecimiento de las colecciones de las bibliotecas académicas, disponiendo la entrega de copias a las bibliotecas universitarias y a la Biblioteca Real. La censura se efectuaba antes de la publicación y la vigilancia la realizaban las autoridades de licencias, no las bibliotecas.

El incumplimiento de la entrega de libros durante estos años fue enorme ${ }^{18}$. En mayo de 1695, la obligación de depósito legal cesó. Desde 1695 hasta 1709, los editores no tuvieron la obligación legal de entregar copias gratuitas y las pocas copias recibidas por las bibliotecas se debieron a la entrega voluntaria ${ }^{19}$. El depósito legal se convirtió en legislación permanente en Inglaterra con el surgimiento de los derechos de autor (copyright).

11 Michael Mendle, "De facto freedom, de facto authority: Press and Parliament, 1640-1643”, The Historical Journal, vol. 38, n.o 2, 1995, pp. 307-332.

12 A Decree of Star-Chamber concerning Printing, 11 July 1637, section xxxirI.

13 Ibíd.

14 Philip, Ian (1983) p. 29. En 1650, la biblioteca de la Universidad de Cambridge comenzó a recibir una copia de todas las obras registradas en la imprenta de Cambridge. McKitterick, David, A History of Cambridge University Press, Volume One Printing and the Book Trade in Cambridge, 1534-1698, Cambridge University Press, Cambridge, 1992 , pp. 261 y 307.

15 BARRington (1938) p. 30.

16 An Act for Preventing Abuses in Printing Seditious, Treasonable, and Unlicensed Books and Pamphlets, and for Regulating of Printing and Printing Presses, 1662, $3 \&$ 4 Car.iI, c.33.

17 BARrington (1938) p. 31.

18 Philip, The Bodleian Library in the Seventeenth of Eighteenth Centuries, cit., p. 52.

19 BarRington (1938) p. 23. 


\section{LA LEY DE COPYRIGHT Y EL DEPÓSITO LEGAL (I7IO-I8O5)}

El Estatuto de 1709 de la reina Ana fue la primera ley de derechos de autor. La industria editorial, representada por la Compañía de Impresores, tuvo un rol determinante en el surgimiento del derecho de autor y sus desarrollos ${ }^{20}$. El acuerdo de 1610 entre Bodley y la Compañía de Impresores ha sido considerado como una de las fuentes de la legislación de copyright de $1709^{[21]}$. Este acuerdo indicó expresamente que uno de sus principales motivos fue "the advancement of good learning"22. La legislación de copyright de 1709 tuvo como objetivo "the encouragement of learning" ${ }^{23}$. Investigaciones recientes señalan que esta primera ley de propiedad intelectual se centró en promover la educación y el interés público. Como afirma Deazley:

... el derecho de autor estaba fundamentalmente relacionado con el público lector, con el fomento y la difusión de la educación, y con la producción continua de libros útiles. Al asignar el derecho a publicar exclusivamente una obra literaria determinada, los parlamentarios del siglo xviII no se ocupaban principalmente de los derechos del individuo, sino que actuaban en el fomento de estos objetivos sociales mucho más amplios $^{24}$.

La ley de derechos de autor estableció, por razones de utilidad pública, la obligación de los impresores de entregar nueve copias de sus publicaciones con destino a las bibliotecas Real ${ }^{25}$, de las universidades de Oxford y Cambridge, de las cuatro universidades de Escocia (Edinburgh, Glasgow, St. Andrews y Aberdeen), del Sion College en Londres y de la Facultad de Abogados en Edinburgo.

Cada impresor debía entregar las nueve copias a la Compañía de Impresores y esta tenía la responsabilidad de distribuir las copias a las bibliotecas. Las sanciones por incumplimiento podrían imponerse, según el caso, al impresor o bien a la Compañía por no remitir las $\operatorname{copias}^{26}$. El incumplimiento del depósito tenía

20 Bently, Lionel y Brad Sherman, The Making of Modern Intellectual Property, Cambridge University Press, 1999, p. 12.

21 Aylmer, Ursula, A Bodleian Library Anthology, Most Noble Bodley!, Bodleian Library, Oxford, 2002, p. 78.

22 A Deed of Grant from the Stationer's Company, entitling the Bodleian Library to the first correct impression of every book printed by them, 1610 .

23 Statute of Anne, 8 Anne, c. 19 (1710), London.

24 Deazley, Ronan, On the Origin of the Right to Copy, Hart Publishing, Oxford, 2004 , p. 226.

25 El Museo Británico fue creado en 1753, y en 1757 George II incorporó la Biblioteca Real dentro del mismo, con copias destinadas a la Biblioteca Real trasladadas al nuevo museo. En noviembre de 1757 la Compañía de Impresores permitió al Museo conservar las copias previstas por ley para la Biblioteca Real. Ver Philip Rowland Harris, A History of the British Museum Library 1753-1973, The British Library, London, 1998.

26 OAtes, J. C. T., Studies in English Printing and Libraries, The Pindar Press, London, 1991, p. 251. 
una multa pecuniaria ${ }^{27}$. Esta imposición de entregar nueve copias de cada libro registrado bajo la ley de derechos de autor fue considerada por los editores como extremadamente onerosa.

Los editores interpretaron que los derechos de autor dependían del registro voluntario. Esta interpretación les permitiría evitar el gravoso depósito. Si los impresores no registraban las obras, no tenían que entregar las nueve copias para las bibliotecas. Múltiples editores, especialmente de obras costosas, que además eran aquellas consideradas como las obras adecuadas para estimular el conocimiento, decidieron no obtener los derechos de autor, ya que el derecho de autor en muchos casos no generaba beneficios que compensaran el valor de las nueve copias. Otros editores adoptaron una estrategia de registro parcial, depositando solo una parte de la obra, de tal forma que si alguna biblioteca quería la obra completa debía comprarla ${ }^{28}$. Como consecuencia de esta situación, la mayoría de las obras depositadas fueron publicaciones baratas y efímeras, como panfletos, sermones y poemas $^{29}$. En efecto, las primeras publicaciones entregadas por la Compañía de Impresores en 1710 fueron obras triviales, como The amazing wonder, A full and true relation of the dismal condition of one John Sexton o Blew-coat-boy ${ }^{30}$. Después de quince años de la promulgación de la ley de derechos de autor, el bibliotecario de Sion College se quejó de la falta de entrega de obras doctas ${ }^{31}$. El depósito también hizo parte de las obligaciones de los editores que obtuvieron copyright por medio de leyes individuales. Por ejemplo, en 1734 el editor Samuel Buckley obtuvo por medio de una ley los derechos exclusivos por el término de catorce años para la impresión y reimpresión de Histories of Thuanus ${ }^{2}$. Dentro de las obligaciones de Buckley se estableció la de entregar nueve copias a las bibliotecas públicas ${ }^{33}$.

En 1775, luego de la importante sentencia Donaldson v. Beckett ${ }^{34}$, las universidades y las bibliotecas depositarias lograron la promulgación de una ley para asegurar el derecho de autor a perpetuidad para las universidades y confirmar la obligación del depósito legal ${ }^{35}$. De acuerdo con esta ley, los derechos de autor solo se otorgarían si el editor entregaba las nueve copias para las bibliotecas. Sin embargo, en 1798 la sentencia Beckford v. Hood afectó radicalmente el depósito. Allí se decidió que el registro no era obligatorio para obtener derechos de copyright, en otras palabras,

27 Statute of Anne, 8 Anne, c. 19 (1710), London.

28 BARrington (1938) p. 37.

29 Ibíd., p. 38.

30 Ontes, Cambridge University Library. A History from the Beginnings to the Copyright Act of Queen Anne, cit., p. 490.

31 Ibíd., p. 251.

32 Feather, John, Publishing, Piracy and Politics. An Historical Study of Copyright in Britain, Mansell, London, 1994 , p. 80.

33 An Act for granting to Samuel Buckley, Citizen and Stationer of London, the sole Liberty of Printing and Reprinting the Histories of Thuanus, with Additions and Improvements, during the Time therein limited, 1734.

34 Donaldson v. Becket (1774) Hansard, 1st ser., 17 (1774): 953-1003. En esta decisión se estableció que el derecho de copyright no es perpetuo

3515 Geo. 3. c. 53, 1775. 
los derechos de autor existían con independencia del registro. Como consecuencia, el interés de los editores por registrar y depositar libros decayó, lo que se evidencia en que un año antes de esta decisión, en 1797, se depositaron 651 obras, y dos años después de la decisión el número se redujo a 366.

Los derechos de autor estimularon la publicación de libros a finales del siglo $\mathrm{XVIII}^{36}$. Los editores aumentaron la edición de nuevas obras y con ello la cantidad de títulos disponibles en el mercado. Al mismo tiempo, evadieron el depósito gratuito de libros, el cual reducía sus ganancias.

En 1800 el Reino de Gran Bretaña y el Reino de Irlanda se unieron ${ }^{37}$, de tal forma que la ley de derecho autor de 1801 se extendió a Irlanda, luego de lo cual el número de copias que había que depositar benefició a otras dos universidades en Dublín ${ }^{38}$; en efecto, el Trinity College Dublin y el King's Inns Dublin adquirieron el derecho de recibir ejemplares de las publicaciones ${ }^{39}$. Así, para comienzos del siglo XIx, once copias de cada libro debían ser entregadas de forma gratuita para las principales bibliotecas académicas. Esta costosa obligación incrementó la animadversión de los impresores y editores hacia el depósito.

\section{LA “BATALLA DE LOS LIBROS”: I805-I836}

El siglo XIX fue de grandes convulsiones para el desarrollo del depósito legal y el derecho de autor. La "batalla de los libros" ${ }^{40}$ consistió en la polémica pública, durante varias décadas, entre las bibliotecas y los editores. Por un lado, las bibliotecas criticaron el incumplimiento de la entrega de libros y, por otro lado, los editores se quejaron persistentemente del depósito legal como una obligación extremadamente onerosa. La batalla de los libros comenzó en 1805 con el panfleto titulado Consultas y observaciones sobre la biblioteca universitaria, publicado por el abogado Basil Montagu ${ }^{41}$.

Montagu decidió escribir sobre el derecho de las bibliotecas cuando encontró imposible consultar en la Biblioteca de la Universidad de Cambridge una copia del

36 Feather, John, "Publishers and politicians: The remaking of the Law of Copyright in Britain 1775-1842, Part I: Legal deposit and the battle of the library tax" (1988) 24 Publishing History 49, p. 51.

37 An Act for the Union of Great Britain and Ireland 1800.

38 Copyright Act 1801 c. 107. Desde 1739 se había prohibido la importación de libros reimpresos en Irlanda, lo cual cambió con la integración de los reinos. Cfr. Act for the Prohibiting the Importation of Books reprinted abroad and first composed or written and printed in Great Britain and for limiting the Prices of Books, 1739, 12 Geo.II, c.36.

39 Aunque hoy en día Irlanda no es parte del Reino Unido, la Biblioteca del Trinity College en Dublín continúa teniendo el derecho de recibir copias como parte del depósito establecido por la legislación del Reino Unido. Luego de la formación del Estado Libre de Irlanda en 1921, en 1927 se creó legislación recíproca de depósito legal con el Reino Unido. Una copia de los libros impresos en Irlanda va a la Biblioteca Británica (para ese entonces Biblioteca del Museo Británico). The Industrial and Commercial Property (Protection) Act, art. 178.

40 Barrington (1938) p. 46.

41 Montagu, Basil, Enquires and Observations Respecting The University Library, Printed by F. Hodson, Cambridge, 1805 . 
séptimo volumen de Term reports porque los editores no habían entregado la copia a la que estaban obligados. En su publicación, Montagu resaltó que la actitud de los editores al no depositar los libros desafiaba el propósito de la legislación de derecho de autor, y que precisamente este volumen no entregado a la biblioteca contenía la decisión de Beckford v. Hood ${ }^{42}$. Haciendo eco de la preocupación de Montagu, en 1807 Edward Christian, profesor de derecho de Cambridge, publicó otro libelo apoyando a las bibliotecas. Christian argumentó que la obligación de entregar copia de las publicaciones a las bibliotecas estaba en consonancia con los "principios de la justicia moral" 43 . Christian propuso que la Universidad de Cambridge iniciara una acción ante la Corte del Rey (The Court of King's Bench) contra uno de los editores reticentes. Una decisión de la Corte sería la mejor forma para resolver la polémica y conocer si la obligación de los editores era equitativa o no ${ }^{44}$.

En 1808 se presentó un proyecto de ley en la Cámara de los Comunes que confirmaba el depósito legal de once copias de todos los impresos, pero fue rechazado porque se consideró que estas disposiciones no eran útiles para los editores ni para las bibliotecas. Los editores argumentaron que una medida tan onerosa constituía una violación de la propiedad privada y, por otro lado, las bibliotecas académicas vieron innecesario recibir un sinnúmero de impresiones frívolas ${ }^{45}$. Las bibliotecas universitarias solo estaban interesadas en las publicaciones doctas.

A finales de 1810, la Universidad de Cambridge decidió iniciar una campaña para el cumplimiento del depósito ${ }^{46}$. En enero de 1811, en diversos periódicos se publicó un "Aviso a los impresores" en el que se advertía que la universidad ordenaría de inmediato iniciar procedimientos legales, en nombre del canciller, maestros y académicos, contra los impresores o editores que después del $1 .^{\circ}$ de febrero de 1811 no entregaran copia de libros publicados a la biblioteca de la universidad como lo disponía la ley ${ }^{47}$. En abril, la universidad solicitó al impresor de Travels in the South of Spain, in letters written a.D. 1809 and 1810 que entregara copia del libro a la biblioteca. El impresor se negó y en mayo se informó a la universidad que el sector editorial estaba recibiendo asesoramiento legal sobre el tema ${ }^{48}$. Finalmente, a mediados de 1811 la Universidad de Cambridge, siguiendo la idea de Christian, presentó una acción contra los impresores.

42 Montagu resaltó que en Beckford $v$. Hood los autores podían iniciar acciones legales por los daños causados cuando una obra era reproducida ilegalmente aun sin que hubiera sido registrada ante la Compañía de Impresores.

43 Christian, Edward, A Vindication of the Right of the Universities of Grep. Britain to a Copy of Every New Publication, University Press, Cambridge, 1807, p. 5.

44 Ibíd., p. 15.

45 Feather, John, "Publishers and politicians: The remaking of the Law of Copyright in Britain 1775-1842, Part I: Legal Deposit and the Battle of the Library Tax" (1988) 24 Publishing History 49, p. 54.

46 Ontes, J. C. T., "Cambridge University and the Reform of the Copyright Act, 1805-1813" (1972) 4 The Library 275, p. 281.

47 The London Gazette, January 151811.

48 OAtes, ob. cit., p. 282. 
La Universidad de Cambridge demandó a Henry Bryer por no haber entregado una copia del libro titulado $A$ vindication of $M r$. Fox's history of the early part of the Reign of James Second ${ }^{49}$. Este libro no se registró ante la Compañía de Impresores y el editor no entregó ninguna de las once copias establecidas en la ley. El demandado argumentó que, al no haber registrado la obra, no tenía la obligación de entregar copias a las bibliotecas. En 1812, en Universidad de Cambridge v. Bryer, la Corte impuso una multa pecuniaria al editor ${ }^{50}$. En su sentencia, lord Ellenborough afirmó que la ley de derechos de autor tenía dos objetivos: proteger los derechos de copyright y favorecer el avance del conocimiento. El propósito del registro y el depósito legal eran diferentes, en ningún caso el derecho de las universidades a recibir libros dependía del registro. La Corte llegó a esta conclusión tras examinar el propósito del depósito legal dentro de la ley de derechos de autor. Para Ellenborough, el depósito legal de libros se centraba en el avance del conocimiento y la literatura, y "el objetivo de la legislación de derechos de autor fue facilitar el acceso al conocimiento" 51 , como lo demuestra la interpretación de los artículos 4 y 5 de la ley. El objetivo de la obligación de entregar once libros era asegurar unas colecciones accesibles a las personas ilustradas, "para las cuales estos libros podrían tener un precio tan considerable que dificultaría el acceso para los intelectuales de medios económicos ordinarios" 52 . Además, la decisión estableció que las disposiciones por las que se establecía la entrega de ejemplares a las bibliotecas universitarias tenían como motivo "que el conocimiento avanzara al estar estas bibliotecas abastecidas constantemente de libros" 53 .

Este caso es importante por varias razones: 1) fue la primera decisión judicial que interpretó la provisión del depósito legal y la relación con la legislación de derecho de autor; 2) analizó el objetivo del depósito legal;3) después de este juicio, el cumplimiento del depósito y el registro de las obras comenzó a aumentar: 271 obras en 1812, 350 en 1813 y 541 en $1814^{[54]}$, y 4) generó una reacción en la esfera pública. En diciembre de 1812, después de la sentencia Universidad de Cambridge v. Bryer, los editores incrementaron la presión para modificar la legislación de derechos de autor. Además, en los años siguientes se publicaron numerosos panfletos y artículos contra la entrega de once copias a las bibliotecas. El depósito legal se convirtió en un tema de atención general por parte de las revistas literarias y uno de los puntos más discutidos en la doctrina de la propiedad literaria durante la primera mitad del siglo XIX.

La duración de la protección de derechos de autor y la obligación del depósito fueron los dos puntos de discusión durante las reformas de copyright en los años

49 Para más detalles de este caso, cfr. ibíd., pp. 282-283.

50 U. Cambridge v. Bryer 1812.

51 U. Cambridge v. Bryer 1812.

$52 U$. Cambridge v. Bryer 1812.

53 U. Cambridge v. Bryer 1812.

54 Oates, J. C. T., "Cambridge University and the Reform of the Copyright Act, 1805-1813" (1972) 4 The Library 275, p. 284. 
siguientes. Los impresores y los editores argumentaron en el parlamento que el depósito implicaba un costo importante para la publicación. Durante el lobby, los editores también solicitaron una extensión del término de protección de derechos de autor. En el debate en la Cámara de los Comunes, en 1813, los editores argumentaron que el depósito de once copias afectaba especialmente la impresión de libros caros y ediciones limitadas ${ }^{55}$. Los editores intentaron demostrar que este oneroso depósito era contrario al fomento del aprendizaje y el conocimiento, ya que los libros más eruditos eran los más costosos, de mayor riesgo económico de publicar e impresos en pequeñas cantidades. El informe del comité en la Cámara de los Comunes concluyó que los derechos de autor debían extenderse a 28 años y el depósito de once copias debía permanecer porque "la continuación de la entrega de todas las obras nuevas, y en ciertos casos, de ediciones posteriores, a las bibliotecas que ahora tienen derecho a recibirlas, tenderá al avance del aprendizaje y a la difusión del conocimiento, sin imponer una carga considerable a los autores, impresores o editores de tales obras" 56 .

A partir de 1813 arreció la publicación de folletos por parte de los editores en contra del depósito. El depósito nuevamente fue tildado de ser un impuesto y un ataque a la propiedad privada. A su vez, Montagu y Christian volvieron a publicar sus defensas a favor de la entrega de copias a las bibliotecas universitarias. En 1813, los editores propusieron ante el comité de derechos de autor de la Cámara de los Comunes enmendar el depósito legal y modificar el alcance de Universidad de Cambridge v. Bryer ${ }^{57}$. Plantearon reducir a cinco el número de copias para las bibliotecas, que las bibliotecas pagaran al menos un cuarto del precio de la publicación y que periódicos, canciones, música y obras sin derechos de autor no deberían ser entregadas ${ }^{58}$. Los editores presentaron evidencia económica sobre la industria editorial, argumentando que el depósito de once copias desalentaba la publicación de los libros menos rentables, aunque finalmente admitieron que aun cumpliendo con la obligación del depósito podrían imprimir todas las ediciones ${ }^{59}$. El sector editorial enfatizó que la duración del copyright debía extenderse a 28 años, pero, al mismo tiempo, algunos editores declararon que la extensión de los derechos de autor no era de gran interés porque no parecía ser útil.

Los defensores del depósito legal, incluyendo algunos editores, consideraron la entrega de libros a las bibliotecas como una excelente y barata forma de publicidad. Los mismos editores aceptaron que la presencia de libros en las bibliotecas públicas sirvió en múltiples casos para aumentar las ventas, al incentivar el gusto

55 Alexander, Isabella, Copyright Law and the Public Interest in the Nineteenth Century, Hart, Oxford, 2010, p. 51.

56 Davies, Giddy, "Report on the Acts respecting Copyright", en The Parliamentary Debates from the Year 1803 to the Present Time, vol. xxvi, T.C. Hansard, London, 1813, p. 709.

57 FeATHER (1988) p. 55.

58 BARRINGTON (1938) p. 53.

59 FEATHER (1988) p. 56. 
por la lectura ${ }^{60}$. Sin embargo, durante todos estos años los editores señalaron que en las bibliotecas depositarias el acceso estaba restringido a un pequeño número de académicos, por lo que no prestaban un servicio al público ${ }^{61}$. Además, la publicidad de nuevas obras a través de las bibliotecas privilegiadas se reducía a unas pocas personas.

Los editores argumentaron que la entrega de libros era un "impuesto" injusto y denunciaron que las bibliotecas vendían muchos de los libros, por lo que deberían pagar por los ejemplares que recibían. La Universidad de St. Andrews negó haber vendido libros recibidos por depósito legal ${ }^{62}$, pero ciertamente la Universidad de Cambridge y otras bibliotecas solían vender los libros que no consideraban útiles $^{63}$. Al mismo tiempo, los editores alegaban que las bibliotecas no tenían la infraestructura para guardar todas las impresiones ${ }^{64}$.

La Universidad de Glasgow defendió intensamente la necesidad de recibir libros de acuerdo con la ley de derechos de autor, especialmente libros costosos. La universidad afirmó que se debía recordar que varias áreas de la educación liberal no se podían llevar a cabo con éxito sin una gran cantidad de libros impresos en ediciones finas, no por mera decoración, sino por el propósito más importante de ilustrar los temas que trataban ${ }^{65}$. Además, la Universidad de Glasgow señaló que el deber para el editor era mínimo en comparación con el importante beneficio para la nación de preservar su patrimonio cultural ${ }^{66}$. En 1814 se promulgó una nueva ley de derecho de autor que mantuvo la entrega de libros a once bibliotecas. La ley extendió el alcance del depósito a libros ilustrados, añadió la obligación de entregar a las bibliotecas copia de los mapas y otros impresos anexos a los libros, y extendió la protección de los derechos de autor con un cambio sustancial. Fue la primera vez en el Reino Unido que la ley de derechos de autor estableció la duración de la vida del autor como el criterio para determinar el tiempo de protección. Deazley afirma que esta enmienda parece ser menos el resultado de las discusiones legislativas que la acción oportunista de último minuto del parlamentario S. E. Brydges ${ }^{67}$.

En cuanto al depósito legal, la nueva ley fue decepcionante para los editores ${ }^{68}$. En 1816, las bibliotecas comenzaron a trabajar juntas para lograr el cumplimiento

60 Minutes of Evidence Taken Before the Select Committee on the Copyright Acts of 8 Anne, C. 19; 15 Geo. III, C. 53; 41 Geo. III, C. 107; and 54 Geo. III, C. 116. (House of Commons Sessional Papers, 1818).

61 Edwards, Edward, A Letter to Benjamin Hawes, Esq M.P Bring Strictures on the "Minutes of Evidence" Taken Before the Select Committee on the British Museum, Effingham Wilson, Royal Exchange, London, 1836, p. 22.

62 Alexander, Isabella, Copyright Law and the Public Interest in the Nineteenth Century, Hart, Oxford, 2010, p. 54.

63 Oates, J. C. T. "Cambridge University and the Reform of the Copyright Act, 1805-1813" (1972) 4 The Library 275, p. 286.

64 Feather (1988) p. 57.

65 BARRINGTON (1938) p. 127.

66 FeATHer (1988) p. 58.

67 Deazley, R., "Commentary on Copyright Act 1814", en Primary Sources on Copyright (1450-1900), L. Bently y M. Kretschmer (eds.), 2008, available at: www.copyrighthistory.org 68 FeATher (1988) p. 59. 
del depósito. El registro de obras creció de 1.235 libros en 1811-1814 a 4.353 en $1815-1818^{[69]}$. Las bibliotecas pidieron todos los libros publicados, incluidos los costosos y las obras por suscripción ${ }^{70}$. Esta situación exacerbó la polémica sobre el depósito, el cual volvió a la esfera pública con un álgido debate en los círculos literarios y políticos.

El ex parlamentario Samuel Egerton Brydges, dueño de una imprenta, se convirtió en el más apasionado representante de los editores contra el depósito legal durante 1817 y 1818 . Brydges y los editores declararon que la entrega de libros era un "impuesto" que constituía un ataque a la libertad de prensa, a la propiedad privada y al libre comercio por ser una carga que tenía como efecto restringir la libre circulación de impresos ${ }^{71}$. Brydges indicó que el depósito de once copias desestimulaba el aprendizaje y restringía la impresión de las obras más costosas y eruditas, y propuso que las bibliotecas debían pagar una parte del precio de las copias, señalando los altos costos de la publicación de obras doctas que se encontraban en proceso de impresión ${ }^{72}$. Además, se discutió acerca de los criterios para seleccionar las obras que serían preservadas por las bibliotecas, pues solo las obras eruditas eran del interés de las bibliotecas, mientras que no era oportuno que fueran depositadas publicaciones que se consideraban triviales. Fue así como las bibliotecas de Cambridge y de Oxford rechazaron las obras de Byron, Beethoven, Wordsworth y Jane Austen ${ }^{73}$. También fue motivo de polémica la colección en las bibliotecas de impresiones inmorales y blasfemas ${ }^{74}$.

En 1818, Brydges redactó un proyecto de ley para modificar el depósito legal. El proyecto no encontró apoyo en el parlamento, pero se nombró un comité para estudiar la propuesta ${ }^{75}$. Las conclusiones del comité estuvieron de acuerdo con los editores. Se sugirió mantener la entrega de copias para el Museo Británico y para las bibliotecas de las universidades de Oxford, Cambridge, Edimburgo y Dublín, y asignar a las demás bibliotecas un fondo para la compra de ejemplares, además de que los libros sin derechos de autor estuvieran exentos de cualquier depósito ${ }^{76}$.

Uno de los temas más polémicos fue la exigencia de las bibliotecas de entregar obras publicadas mediante suscripción. Las bibliotecas detuvieron la suscripción a múltiples publicaciones costosas y exigieron las once copias gratuitamente. En varios casos los editores argumentaron que esta práctica disuadió la publicación y la finalización de obras. Problemas específicos con el depósito de obras comer-

69350 copias en 1813, 541 en 1814 y 1.244 en 1815. McKitterick, David, A History of Cambridge University Press, Volume One, Printing and the Book Trade in Cambridge, 1534-1698, Cambridge University Press, Cambridge, 1992, p. 414.

70 BarRington (1938) p. 63.

71 Feather (1988) p. 62.

72 Ibid. p. 63.

73 Barrington (1938) pp. 69, 71, 73.

74 Alexander (2010) p. 58.

75 Brydges, Samuel Egerton, Four Tracts on Copyright 1817-1818, Garland Publishing, London, 1974.

76 Barrington (1938) pp. 69-70. 
cializadas por suscripción fueron expuestos durante estos años. Según William Daniell, uno de los primeros grabadores a color, la finalización de su obra titulada Voyage around Great Britain, publicada por Longmans, podría detenerse debido al depósito ${ }^{77}$. Longman se negó a publicar otros proyectos, como la obra del barón de Humboldt sobre las plantas no descritas de América del Sur ${ }^{78}$. Samuel Lysons, otro editor de obras eruditas, expresó en 1818 su frustración porque después de 1814 las bibliotecas retiraron la suscripción a la edición limitada de solo 50 copias de su Reliquae Britannico-Romano y comenzaron a exigir copias gratuitas. Lysons sugirió la impresión en el extranjero como una solución para evitar el depósito ${ }^{79}$. Los editores se negaron a entregar copias de ediciones limitadas y el Museo Británico decidió iniciar una acción legal.

El Museo Británico exigió la entrega de la primera parte del quinto volumen de la muy limitada edición de la Flora Groeca, uno de los libros sobre botánica más costosos jamás publicado. Para tener una idea del valor de esta publicación, cuando los diez volúmenes de la Flora Groca finalmente se completaron en 1840, el costo de cada copia ascendió a aproximadamente $£ 620$, una suma astronómica teniendo en cuenta que el salario medio anual para un varón adulto en Inglaterra y Gales era de aproximadamente $£ 36^{[80]}$. Este trabajo comenzó a ser publicado en 1806 y continuó con intervalos gracias a los fondos testamentarios del Dr. Sibthorpe de Oxford, quien en vida preparó esta publicación. Solo se realizaron 30 copias impresas de esta lujosa obra, la cual tuvo 26 suscriptores. Significativamente, las bibliotecas de Oxford y Cambridge estaban entre los suscriptores ${ }^{81}$. La publicación se realizó a pérdida ya que el costo de la edición fue más alto que el precio de venta de las copias ${ }^{82}$.

El Museo Británico también demandó al editor Payne al no recibir la copia gratuita solicitada. En 1826, la decisión de British Museum v. Payne, que fue confirmada en 1828, resolvió la polémica sobre el depósito de esta clase de obras publicadas por suscripción. El caso se trató de resolver si una parte de una publicación periódica o editada por volúmenes debía ser entregada a las bibliotecas bajo la obligación de la ley de derechos de autor, y la decisión estableció que la obligación solo recaía sobre libros o volúmenes completos, basándose en dos argumentos referidos a la hermenéutica de la norma de depósito legal. En primer lugar, se dijo, las palabras de la ley deben interpretarse en su sentido natural y con su acepción habitual; en este orden de ideas, una parte de un volumen no es un libro ni un volumen. En segundo lugar, se afirmó, las sanciones deben tener una

77 Feather (1988) p. 64.

78 Parsons, IAn, "Copyright and Society", en Asa, Brigs, Essays in the History of Publishing, In Celebration of the 250th Anniversary of the House of Longman 1724-1974, Longman, London, 1974, p. 46.

79 Feather (1988) p. 64.

80 Harris, Stephen, The Magnificent Flora Graeca. How the Mediterranean came to the English Garden, Bodleian Library, Oxford, 2007, p. 163.

81 The New Monthly Magazine and Literary Journal, 1827, Feb 1, p. 64.

82 The Trustees of the British Musum v. Payne and Foss, p. 878. 
interpretación restringida ${ }^{83}$. Este fue un triunfo muy importante para los editores, que fue anunciado por los medios de comunicación como una victoria contra la "monstruosa Ley de Copyright" ${ }^{44}$. De forma errónea, en 1828, se publicitó que la demanda la habían ganado los editores debido a que el depósito legal no aplicaba a las publicaciones que no tenían amplia circulación ${ }^{85}$. Toda esta propaganda de los medios de comunicación y los editores ayudó a crear el ambiente para una nueva reforma de la ley de derechos de autor.

En 1831, el informe de la Comisión Real de Investigación de las universidades de Escocia recomendó que las bibliotecas de Escocia recibieran una compensación pecuniaria en lugar de los libros por depósito legal ${ }^{86}$. Con este nuevo enfoque, en 1836, James Silk Buckingham, un reformador que apoyó la abolición de todo tipo de restricciones a la libertad de prensa y la circulación de impresos ${ }^{87}$, introdujo un proyecto de ley que eliminaba la entrega gratuita de las obras y establecía un fondo económico para cada biblioteca. En sus argumentos, Buckingham se refirió al depósito legal como un impuesto ("copy tax") y conectó la discusión con otros debates parlamentarios, como el del llamado impuesto sobre el conocimiento ${ }^{88}$. El Museo Británico, las universidades de Oxford y Cambridge, la Facultad de Abogados y el Trinity College rechazaron esta propuesta, manifestando que no querían cambiar la entrega de los libros por dinero adicional para adquisiciones ${ }^{89}$. El Museo Británico afirmó que el depósito legal era esencial para su misión de recaudar todas las publicaciones, incluyendo reimpresiones con pequeñas adiciones. Al final del debate parlamentario, la nueva ley de copyright de 1836 estableció que estas bibliotecas mantendrían el derecho a recibir copias de libros y las demás bibliotecas recibirían una suma anual para la compra de publicaciones. Este cambio fue parte de una era de reformas institucionales en el Imperio Británico ${ }^{90}$.

La ley de derechos de autor de 1838 ofreció protección a las obras británicas publicadas en el extranjero y a las obras extranjeras publicadas en el Imperio Británico ${ }^{91}$. Como requisito para la protección, la ley exigía el registro y el depósito de la obra en el Stationer's Hall, copia que debía ser enviada al Museo Británico ${ }^{92}$. Pero fue solo con la ley de copyright de 1842 que el depósito legal se hizo obligatorio para todo el Imperio Británico. El artículo 6 estableció que copia de todos los libros publicados en los dominios británicos tendría que ser entregada dentro de los doce meses después de su publicación ${ }^{93}$. Hubo múltiples dificultades para

83 Ibíd. (148 E.R. 876 p. 880).

84 The New Monthly Magazine and Literary Journal, January, 1827, p. 64.

85 The Gentleman's Magazine, February, 1828, p. 170.

86 Alexander (2010) p. 61.

87 Feather (1988) p. 68.

88 Principalmente el impuesto al papel. H. Dagnall, "The taxes on knowledge: Excise duty on paper", The Library, vol. 6-xx, n. ${ }^{4}$, 1998.

89 BARRINGTON (1938) p. 76.

90 Feather (1988) p. 69.

91 International Copyright Act (1838).

92 Barrington (1938) p. 78.

93 The Copyright Act 1842 (5 \& 6 Vict. c. 45). 
el cumplimiento de esta legislación, ya que no existían los medios para detectar nuevas publicaciones en los dominios, los editores rechazaban la obligación o simplemente la ignoraban, y el envío de los libros era costoso y difícil ${ }^{94}$.

Desde 1868 fue claro que el derecho de autor surgía de manera independiente del depósito legal y el registro. En 1868, en el caso Routledge v. Low, la Corte declaró que la obligación de entregar una copia al Museo Británico de cada libro publicado en cualquier lugar bajo el dominio británico era independiente de la existencia o inexistencia de los derechos de copyright en el libro ${ }^{95}$.

En la segunda mitad del siglo xix, el depósito legal se consolidó como una medida eficaz para la recolección de la producción bibliográfica nacional del Reino Unido. La legislación de copyright de 1842, y luego de 1911, mantuvo la obligación de entregar copia de cada libro publicado al Museo Británico, así como el derecho de las otras cuatro bibliotecas para exigir también una copia de las publicaciones ${ }^{96}$. Bajo la dirección de Antonio Panizzi (1797-1879), la biblioteca del Museo Británico se convirtió en la mayor biblioteca del mundo ${ }^{97}$. Panizzi reorganizó y modernizó sus servicios, instituyó una campaña eficaz para hacer cumplir el depósito, creó reglas de catalogación para la organización de los materiales de la biblioteca e implementó un robusto sistema de intercambios y compras internacionales ${ }^{98}$.

\section{PATRIMONIO NACIONAL Y DEPÓSITO LEGAL}

Durante el siglo xx, las colecciones de la biblioteca del Museo Británico, incluyendo las colecciones formadas por el depósito legal, empezaron a ser administradas y protegidas como patrimonio documental. En 1915 se reguló el depósito legal, excluyendo de su ámbito los anuncios comerciales o cualquier otro material listado por la Junta de Comercio ${ }^{99}$. Además, se reconoció que todos los materiales recibidos debían ser preservados como parte del patrimonio nacional, prohibiendo a la biblioteca deshacerse de las publicaciones. Esta fue una situación muy diferente de la anterior operación de depósito legal. Durante los siglos anteriores, las bibliotecas depositarias vendieron muchos de los libros que consideraron como no útiles o no dignos de preservación ${ }^{100}$. Las nuevas políticas patrimoniales del siglo

94 Barrington (1938) p. 147.

95 Routledge $v$. Low 1868.

96 S viI, Copyright Law Amendment Act, 1842, 5 \& 6 Vict., c. 45) s 15, Copyright Act 1911 .

97 Edward Miller, Prince of Librarians: The Life \& Times of Antonio Panizzi of the British Museum, The Ohio University Press, Ohio, 1967.

98 Ilse Sternberg, "Policies for the acquisition of printed books at the British Museum Library, 1837-1960: With particular attention to the procurement of works from Africa, Asia, the Caribbean and Latin America”, Ph.D. Thesis, University College, London, 1991. 99 Copyright (British Museum) Act 1915.

100 Por ejemplo, desde 1751 la Universidad de Cambridge autorizo vender los libros recibidos por depósito legal. OAtes, J. C. T., Studies in English Printing and Libraries, The Pindar Press, London, 1991, p. 253. Ver también OAtes, J. C. T., "Cambridge University and the reform of the Copyright Act, 1805-1813" (1972) 4 The Library 275, p. 286. 
xx impusieron obligaciones y deberes de conservación. Estas primeras discusiones políticas de conservación en relación con el patrimonio documental coincidieron con las primeras legislaciones de patrimonio cultural en el Reino Unido para la protección de monumentos y del medio ambiente.

Después de la Primera Guerra Mundial, el Museo Británico sufrió para hospedar las crecientes colecciones documentales formadas por el depósito legal. Esto creó un debate en dos direcciones; por un lado, una mayor libertad para que el Museo Británico rechazara material y, por otro lado, la desintegración de las colecciones. En 1930, la Comisión Real de Galerías del Museo Nacional se opuso a la idea de cualquier desintegración de las colecciones de la Biblioteca Nacional ${ }^{101}$. Esta posición estuvo en consonancia con la integridad de las colecciones como parte de la protección del patrimonio, pues la integridad de una colección está conectada a su valor nacional en su totalidad, más que el valor individual de los diferentes objetos que la forman ${ }^{102}$.

El depósito legal hizo parte de las políticas de colecciones, con la adopción de criterios para la selección del material considerado importante para las generaciones futuras. En 1932, la legislación del Museo Británico eliminó la obligación de entrega de material de menor importancia y difícil de manejar. Se facultó a la Biblioteca para recibir material efímero como anuncios comerciales, calendarios y hojas de pared solo bajo la solicitud del Museo ${ }^{103}$. En 1968, la Ley de Teatros hizo obligatoria la entrega de guiones teatrales al Museo Británico ${ }^{104}$.

En 1973 se creó formalmente la Biblioteca Británica ${ }^{105}$ y los tres departamentos de la biblioteca del Museo Británico se fusionaron dentro de la nueva institución ${ }^{106}$. En la década de 1990, la biblioteca consolidó un conjunto de reformas para modernizar sus servicios; se trasladó a un nuevo edificio ${ }^{107}$ y comenzó a investigar el depósito legal de material no impreso ${ }^{108}$. En la misma década, el número de documentos digitales distribuidos en soportes como discos compactos (CD) y disquetes aumentó significativamente. Nuevas obras digitales, como videojuegos, software y obras multimedia, se volvieron de uso diario y generalizado. Además, la enorme cantidad de información publicada en internet de importancia histórica

101 Royal Commission on National Museums and Galleries (1930), p. 83, cit. por BARRINGTON (1938) p. 122.

102 Por ejemplo, el Consejo de las Artes de Inglaterra designa colecciones de museos, bibliotecas y archivos de importancia nacional que "existen para identificar y celebrar colecciones de resonancia destacada que dejan en claro nuestra comprensión del mundo y que significa ser humano". Arts Council England, The Designation Scheme, Guidance for Applicants, Arts Council England, Birmingham, 2015.

103 British Museum Act 1932, Schedule.

104 Theatres Act 1968, S 11.

105 British Library Act 1972. En 1958 el gobierno comenzó a discutir la creación de un nuevo edificio para la biblioteca; ver Philip Rowland Harris, A History of the British Museum Library 1753-1973, The British Library, London, 1998, p. 673.

106 Philip Rowland Harris, A History of the British Museum Library 1753-1973, The British Library, London, 1998, p. xiv.

107 The British Library at St. Pancras, Library Management (1998) vol. 19, 6.

108 Report of the Working Party on Legal Deposit, British Library, London, 1998. 
hizo necesaria una reforma legislativa para la recolección y preservación del patrimonio cultural digital. Durante el siglo xx, el depósito legal en el Reino Unido solo cubrió material impreso. Las publicaciones audiovisuales, material fonográfico y otros nuevos medios de comunicación, como la información digital, estuvieron fuera del alcance de la ley.

En el año 2003 se promulgó la Ley de Depósito Legal, la cual extendió el depósito a publicaciones en formatos diferentes al impreso ${ }^{109}$. Con ello, después de casi tres siglos, se eliminaron las disposiciones de depósito legal de la legislación de derechos de autor ${ }^{110}$. Sin embargo, como ya lo hemos señalado en otras oportunidades, la relación del derecho de autor y el depósito legal en el ambiente digital es ahora mucho más estrecha de lo que lo era en el mundo de la cultura impresa, especialmente con la creación de archivos de publicaciones web. La Ley de Depósito Legal y su regulación de $2013^{[111]}$ permiten a la Biblioteca Británica, las bibliotecas nacionales de Escocia y de Gales, la Biblioteca Bodliana de la Universidad de Oxford, la Biblioteca de la Universidad de Cambridge y la Biblioteca del Trinity College en Dublín realizar copias de las páginas web publicadas en el Reino Unido sin necesidad de solicitar autorización a los titulares de derechos de autor. Además, la Ley de Depósito Legal y su regulación crearon una serie de excepciones al derecho de autor que permiten el acceso y preservación del patrimonio cultural digital del Reino Unido.

\section{CONCLUSIÓN}

Tanto el derecho de autor como el depósito legal han sido mecanismos para promover el avance y acceso al conocimiento. Ambas instituciones jurídicas han compartido un recorrido histórico ya que son complementarias. El depósito legal ha sido una evidente faceta del interés público que subyace a los derechos de autor. El recuento histórico de estas dos figuras legales nos muestra que el depósito legal es una institución exógena, pero complementaria del derecho de autor, en cuanto a políticas públicas de regulación de la información, el conocimiento y las industrias culturales.

La relación histórica entre el depósito legal y el derecho de autor en la legislación del Reino Unido ha sido interpretada de múltiples formas. Para John Feather la inclusión de depósito legal en la ley de derechos de autor fue un "accidente" ${ }^{112}$; para Kenneth Crews, el depósito legal fue un requisito para la protección del derecho de autor ${ }^{113}$; para Isabella Alexander, fue parte del interés público de los derechos

109 Legal Deposit Libraries Act 2003, art. 6.

110 Legal Deposit Libraries Act 2003.

111 The Legal Deposit Libraries (Non-Print Works) Regulations 2013.

112 Feather, John, Publishing, Piracy and Politics. An Historical Study of Copyright in Britain, Mansell, London, 1994, p. 7.

113 Crews, Kenneth D., "Legal deposit in four countries: Laws and library services" (1988) 80 Law Libr J 551, p. 565. 
de autor ${ }^{114}$; para Peter Menell, esta relación estuvo basada en un tema probatorio de la existencia derechos de autor ${ }^{115}$. Con una posición más radical, el ilustre profesor de Oxford, William Searle Holdsworth, escribió que el depósito legal no tiene ninguna conexión lógica con los derechos de autor ${ }^{116}$. En contraste, para Frederick William Ratcliffe, quien fue director de la biblioteca de la Universidad de Cambridge, ambos temas están relacionados, pero la inclusión del depósito legal en la ley de derechos de autor ha oscurecido su importancia cultural ${ }^{117}$.

En el Reino Unido, la Compañía de Impresores y los editores en general, a regañadientes, se vieron obligados a entregar copias de sus publicaciones a las bibliotecas porque la ley lo exigió y se tomaron acciones judiciales para su cumplimiento. Esto nos muestra que la acción estatal y el lobby de las universidades de investigación y de las principales instituciones de la memoria (como las bibliotecas, los archivos y los museos nacionales) tienen un papel fundamental para impulsar acciones legislativas en contextos donde las industrias culturales tienen un gran poder de negociación y poco interés en otra cosa que no sean sus ganancias económicas.

\section{REFERENCIAS}

\section{LEGISLACIÓN}

Act for Granting to Samuel Buckley, Citizen and Stationer of London, the sole Liberty of Printing and Reprinting the Histories of Thuanus, with Additions and Improvements, during the Time therein limited, 1734.

Act for Preventing Abuses in Printing Seditious, Treasonable, and Unlicensed Books and Pamphlets, and for Regulating of Printing and Printing Presses, 1662,3 \& 4 Car.II, c.33.

Act for the Prohibiting the Importation of Books reprinted abroad and first composed or written and printed in Great Britain and for limiting the Prices of Books, 1739, 12 Geo.II, c.36.

Act for the Union of Great Britain and Ireland 1800.

British Library Act 1972

British Museum Act 1932.

Copyright (British Museum) Act 1915.

114 Alexander, Isabella, Copyright Law and the Public Interest in the Nineteenth Century citation style!, Hart, Oxford, 2010, p. 62; Gilchrist, John, "Copyright deposit, legal deposit or library deposit?: The Government's role as preserver of copyright material” (2005) 5 Queensland University of Technology Law \& Justice Journal 177, p. 193.

115 Menell, Peter S., "Copyright in context: Institute for Intellectual Property \& Information Law Symposium: Article: Knowledge Accessibility and Preservation Policy for the Digital Age" (2007) 44 Hous. L. Rev. 1013.

116 W. S. Holdsworth, "Review of Books" (1939) 54 The English Historical Review 156 , p. 156.

117 Ratcliffe, F. W., "Legal deposit: Not a copyright issue, a cultural legacy for the future", Logos, vol. 2, n.o 2 (1991) p. 82. 
Copyright Act 1775, 15 Geo. 3. c. 53.

Copyright Act 1801 c. 107.

Copyright Act 1911.

Copyright Law Amendment Act, 1842, 5 \& 6 Vict., c. 45.

Decree of Star-Chamber concerning Printing, 11 July 1637.

Legal Deposit Libraries Act 2003.

Ordonnance de Montpellier du 28 décembre 1537 (France) (BNF, Ms 22076, Catalogue des Actes de François 1er, N 9476).

Statute of Anne, 8 Anne, c. 19 (1710), London.

The Copyright Act 1842 (5 \& 6 Vict. c. 45).

The Industrial and Commercial Property (Protection) Act 1927 (Irlanda).

The Legal Deposit Libraries (Non-Print Works) Regulations 2013.

Theatres Act 1968.

JURISPRUDENCIA

Donaldson v. Becket (1774) Hansard, 1st ser., 17 (1774): 953-1003.

U. Cambridge v. Bryer 1812.

The Trustees of the British Musum v. Payne and Foss (148 E.R. 876).

Routledge v. Low 1868.

DOCTRINA

Alexander, Isabella. Copyright Law and the Public Interest in the Nineteenth Century, Hart, Oxford, 2010.

Arts Council England, The Designation Scheme, Guidance for Applicants, Arts Council England, Birmingham, 2015.

Aylmer, Ursula. A Bodleian Library Anthology, Most Noble Bodley!, Bodelian Library, Oxford, 2002.

Barrington Partridge, R. C. The History of the Legal Deposit of Books throughout the British Empire, The Library Association, London, 1938.

Bentuy, Lionel y Sherman, Brad. The Making of Modern Intellectual Property, Cambridge University Press, 1999.

Bjerregärd, Estrid. "Legal deposit - Purpose and scope in Modern Society", Libri, Copenaghen, 23 (4), 1973.

Brydges, Samuel Egerton. Four Tracts on Copyright 1817-1818, Garland, London, 1974.

Chastel, André y Babelon, Jean-Pierre. La notion du patrimoine, Paris, Liana Levi, 1980.

Christian, EDward. A Vindication of the Right of the Universities of Great Britain to a Copy of Every New Publication, University Press, Cambridge, 1807. 
Crews, Kenneth D. "Legal deposit in four countries: Laws and library services" (1988) 80 Law Library Journal 551.

Dagnall, H. “The Taxes on Knowledge: Excise Duty on Paper”, The Library, vol. 6-XX, n. ${ }^{\circ}$ 4, 1998, 347.

Deazley, R. "Commentary on Copyright Act 1814", en Primary Sources on Copyright (1450-1900), L. Bently y M. Kretschmer (eds.), 2008, available at: www.copyrighthistory.org

Deazley, Ronan. On the Origin of the Right to Copy, Hart Publishing, Oxford, 2004.

Dougnac, Marie-Thérèse y M. Guilbaud. "Le dépôt légal: son sens et son evolution" (1960) 8 Bulletin des Bibliothèques de France.

FEATHer, John. "Publishers and politicians: The remaking of the Law of Copyright in Britain 1775-1842, Part I: Legal Deposit and the Battle of the Library Tax" (1988) 24 Publishing History 49.

Feather, John. Publishing, Piracy and Politics. An Historical Study of Copyright in Britain, Mansell, London, 1994.

Giddy, Davies. "Report on the Acts Respecting Copyright", en The Parliamentary Debates from the Year 1803 to the Present Time, vol. Xxvi, T.C. Hansard, London, 1813.

Gilchrist, John. "Copyright deposit, legal deposit or library deposit?: The Government's Role as preserver of Copyright Material” (2005) 5 Queensland University of Technology Law \& Justice Journal 177.

Harris, Philip Rowland. A History of the British Museum Library 1753-1973, The British Library, London, 1998.

Harris, Stephen. The Magnificent Flora Graeca. How the Mediterranean came to the English Garden, Bodleian Library, Oxford, 2007.

Holdsworth, W. S. "Review of books" (1939) 54 The English Historical Review 156.

Ian, Philip. The Bodleian Library in the Seventeenth of Eighteenth Centuries, Clarendon Press, Oxford, 1983.

McKitterick, David. A History of Cambridge University Press, Volume One Printing and the Book Trade in Cambridge, 1534-1698, Cambridge University Press, Cambridge, 1992.

Mendle, Michael. "De facto freedom, de facto authority: Press and Parliament, 1640-1643” (1995) 38 (2) The Historical Journal, 307.

Menell, Peter S. "Copyright in context: Institute for Intellectual Property \& Information Law Symposium: Article: Knowledge Accessibility and Preservation Policy for the Digital Age" (2007) 44 Hous. L. Rev. 1013.

Miller, Edward. Prince of Librarians: The Life \& Times of Antonio Panizzi of the British Museum, The Ohio University Press, Ohio, 1967. 
Minutes of Evidence Taken Before the Select Committee on the Copyright Acts of 8 Anne, C. 19; 15 Geo. III, C. 53; 41 Geo. III, C. 107; and 54 Geo. III, C. 116. (House of Commons Sessional Papers, 1818).

Montagu, Basil. Enquires and Observations Respecting The University Library, Printed by F. Hodson, Cambridge, 1805.

Oates, J. C. T. "Cambridge University and the Reform of the Copyright Act, 1805-1813" (1972) 4 The Library 275.

OAtes, J. C. T. Cambridge University Library. A History from the Beginnings to the Copyright Act of Queen Anne, Cambridge University Press, Cambridge, 1986.

Oates, J. C. T. Studies in English Printing and Libraries, The Pindar Press, London, 1991.

Parsons, Ian. "Copyright and Society", en Asa, Brigs, Essays in the History of Publishing, In Celebration of the 250th Anniversary of the House of Longman 1724-1974, Longman, London, 1974.

Principaux Textes sur le Dépôt Légal, Historique, Direction de l'Administration Générale, Paris, 1992.

Ratcliffe, F. W. "Legal deposit: Not a copyright issue, a cultural legacy for the future", Logos, vol. 2, n. ${ }^{\circ} 2$ (1991).

Report of the Working Party on Legal Deposit, British Library, London, 1998.

Sternberg, Ilse. Policies for the Acquisition of Printed Books at the British Museum Library, 1837-1960: With Particular Attention to the Procurement of Works from Africa, Asia, the Caribbean and Latin America, Ph.D. Thesis, University College, London, 1991.

Stiruing, P. et al. "The state of e-legal deposit in France: Looking back at five years of putting new legislation into practice and envisioning the future" (2012) 38 (1) IFLA Journal 5.

The British Library at St. Pancras, Library Management (1998) vol. 19, 6.

The Gentleman's Magazine, February, 1828.

The London Gazette, January 151811.

The New Monthly Magazine and Literary Journal, 1827, Feb 1. 